ANNALS OF

CLINICAL

NEUROPHYSIOLOGY

\section{Transcranial magnetic stimulation parameters as neurophysiological biomarkers in Alzheimer's disease}

\author{
Juyoun Lee, Ae Young Lee \\ Department of Neurology, Chungnam National University Hospital, Daejeon, Korea
}

Transcranial magnetic stimulation (TMS) is a safe and noninvasive tool for investigating the cortical excitability of the human brain and the neurophysiological functions of GABAergic, glutamatergic, and cholinergic neural circuits. Neurophysiological biomarkers based on TMS parameters can provide information on the pathophysiology of dementia, and be used to diagnose Alzheimer's disease and differentiate different types of dementia. This review introduces the basic principles of TMS, TMS devices and stimulating paradigms, several neurophysiological measurements, and the clinical implications of TMS for Alzheimer's disease.

Key words: Transcranial magnetic stimulation; Alzheimer's disease; Diagnosis; Treatment

\section{INTRODUCTION}

Transcranial magnetic stimulation (TMS) is a safe and non-invasive intervention for electrically stimulating neural tissues using magnetic pulses. After Barker et al. ${ }^{1}$ reported that the method could be used to safely stimulate the human brain in 1985, Pascual-Leone et al. ${ }^{2}$ reported that TMS could safely modulate brain activities by applying high-frequency repetitive TMS (rTMS). TMS has been used to investigate cortical excitability and provide additional evidence for use in diagnoses and prognoses. In addition, rTMS has been applied in therapeutic interventions for various neurological and psychiatric diseases. TMS has recently been introduced as a novel approach to diagnosing and differentiating dementia, and to enhance cognitive function in both cognitively normal and impaired patients. ${ }^{3}$ This article reviews the TMS measurements that provide information about the neurophysiological state of Alzheimer's disease (AD) and the future value of TMS in the diagnosis and treatment of AD. 


\section{PRINCIPLES OF TMS}

\section{Action mechanism of TMS}

TMS is based on Faraday's law of electromagnetic induction. ${ }^{4}$ Rapidly changing pulses of current passing through a coil positioned above the head generate magnetic fields that penetrate the scalp and skull to reach the brain and induce secondary electric currents in the cortex. ., $^{6}$ These electric currents depolarize the neuron membranes either directly or indirectly. They can markedly influence changes in excitability from the regional cortex to the subcortex and distant areas that are connected to the stimulated area via neural pathways, ${ }^{7-10}$ with these changes ultimately affecting human cognition and behavior. ${ }^{11}$

rTMS can be used to modulate synaptic plasticity, especially long-term potentiation (LTP) ${ }^{12}$ and long-term depression (LTD). ${ }^{13}$ LTP is a phenomenon in which synapses are persistently strengthened, and it is an important mechanism of memory and learning at the neuron level. High-frequency rTMS and theta-burst stimulation (TBS) affect the expression levels of various receptors, including N-methyl-D-aspartate (NMDA) receptors, ${ }^{14}$ and neurotransmitters. ${ }^{15}$ High-frequency rTMS reduces the synaptic conduction threshold, leading to enhanced synaptic connections and synaptic plasticity. ${ }^{16}$ These structural and functional changes in synapses lead to the induction of LTP, which affects cognitive function.

\section{TMS devices and stimulation paradigms}

A TMS device consists of a coil and a stimulator. There are several types of coil: circular or round, figure-of-eight, double-cone, and $\mathrm{H}$-coil. The amplitude of currents flowing in the coil controls the intensity of the induced magnetic field, and the type of coil determines where that field is focused. A figure-of-eight coil is the most widely used and provides more focal stimulation compared with a circular coil, for which the electric field is more widely distributed and provides bihemispheric stimulation. ${ }^{17}$ An $\mathrm{H}$-coil stimulates a wide and deep area of the brain, ${ }^{18}$ while a double-cone coil stimulates deep and focal areas such as deep parts of the cerebellum ${ }^{19}$ or the leg motor area. ${ }^{20}$

The stimulator generates pulse waves of appropriate frequency, intensity, and number of pulses to influence the effects of TMS. ${ }^{21}$ Generally, high-frequency stimulation $(5-25 \mathrm{~Hz})$ induces cortical excitability while low-frequency stimulation ( $\leq 1 \mathrm{~Hz}$ ) inhibits cortical activities. ${ }^{22}$ However, high-frequency stimulation at low intensities decreases cortical activity, while high-frequency stimulation at high intensities increases cortical excitability. ${ }^{23}$ TBS is a variant of rTMS that increases cortical excitability when applied intermittently and decreases cortical excitability when applied continuously. ${ }^{24}$

\section{TMS OUTCOME MEASURES IN DEMEN-} TIA

\section{Single-pulse TMS}

Single-pulse TMS is generally used to estimate the motor threshold (MT) or cortical excitability when recording motor evoked potentials (MEPs). When single-pulse TMS is applied to the primary motor cortex, series of recordable corticospinal volleys are generated that reflect the trans-synaptic activation of cortical neurons.

\section{Motor threshold}

The MT is related to the integrity of the corticospinal pathway. It is used to characterize each level of excitability along the pathway, or as a reference measure of excitability when determining the intensity to apply in different stimulation protocols. The resting MT (RMT) refers to the lowest TMS intensity that evokes MEPs with a peak-to-peak amplitude of $\geq 50 \mu \mathrm{V}$ in at least $50 \%$ of successive trials in the relaxed target muscle. ${ }^{25}$

RMT is decreased in the early stage of $A D$ and is lowest in the advanced stage. ${ }^{26}$ The reduced RMT in early-stage AD indicates hyperexcitability of the motor cortex, and a relative increase in excitability in the excitatory/inhibitory balance, which is caused by impairment of inhibitory interneuron function. ${ }^{27}$ Decreased RMT following disease progression might be compensatory for the loss of motor cortex neurons in the mild-to-moderate stage of AD. ${ }^{28}$ Mild cognitive impairment (MCl) patients also show a lower RMT than healthy subjects, similar to AD patients. These results suggest that the hyperexcitability emerges from the $\mathrm{MCl}$ state when the cognitive function is relatively good. ${ }^{29}$

\section{Motor evoked potential}

The MEP amplitude reflects the integrity of the corticospinal 
tract and the excitability of the motor cortex, nerve roots, and conduction along the peripheral motor pathway to the muscle. A reduced MEP amplitude reflects the failure of central motor conduction. The small-amplitude MEPs seen in stroke ${ }^{30}$ involve the motor cortex or corticospinal tract, hydrocephalus, and multiple sclerosis. Most studies have found that the MEP amplitude does not differ between AD patients and healthy controls, whereas MEPs are lower in frontotemporal dementia (FTD) patients. This indicates the involvement of the corticospinal tract in the early stage of FTD but not in $A D{ }^{31}$

\section{Contralateral silent period}

Applying suprathreshold TMS to the motor cortex during tonic voluntary contraction of the contralateral muscles results in suppression of the electromyographic activity of those muscles lasting a few hundred milliseconds. ${ }^{32}$ This phenomenon is called the contralateral silent period (CSP), which reflects activation of the inhibitory cortical interneurons, which is mediated by gamma-aminobutyric acid (GABA)-B. ${ }^{33}$ Severe synapse loss with failure of inhibitory control represented as a significant reduction of the CSP in patients with advanced $A D{ }^{34}$

\section{Paired-pulse TMS}

The paired-pulse TMS protocol involves the application of two different sequential stimuli with various interstimulus intervals (ISIs). This can provide information about the integrity of corticocortical connections. There are two inhibitory circuits and two facilitatory circuits determined by the intensity and ISI of this protocol: short-interval intracortical inhibition (SICI), long-interval intracortical inhibition (LICI), intracortical facilitation (ICF), and short-interval intracortical facilitation. Paired-pulse TMS, like short-latency afferent inhibition (SAI), is used to investigate sensory-motor interactions in the cerebral cortex.

\section{$\mathrm{SICl}$ and ICF}

A subthreshold magnetic conditioning stimulus (CS) is followed by a suprathreshold magnetic test stimulus (TS) delivered to the same target area using the same coil. The TS induces a corticospinal output leading to an MEP, while a subthreshold CS only excites local cortical interneurons. Therefore, this stimulation protocol can be used to assess the effects of interneurons on cortical output. ${ }^{35}$ Paired-pulse TMS shows inhibitory intercortical phenomena (with a short ISI of 1-4 ms; SICI) and facilitatory intercortical phenomena (with a longer ISI of > $5 \mathrm{~ms}$; ICF) that are mediated by GABA-A and glutamatergic NMDA, respectively. ${ }^{36}$

\section{Short-latency afferent inhibition}

The SAI protocol involves applying conditioning electric stimulation to the median nerve at the wrist $20-25$ ms before applying a contralateral TMS test pulse, with the timing compatible with the activation of the test pulse alone. SAI is a putative marker of central cholinergic activity and depends on the corticocortical inhibitory and thalamocortical circuits.

$\mathrm{SICl}$ and $\mathrm{SAI}$ are significantly decreased in patients with $\mathrm{AD}$ and $\mathrm{MCl}$, which reflects low cholinergic activity. ${ }^{37}$ Other studies have found SAI to be normal in FTD patients, but reduced in patients with $A D$ and dementia with Lewy bodies (DLB). ${ }^{38}$ DLB patients showed a significant reduction of cholinergic activity as measured by SAl, which was correlated with their hallucinations. ${ }^{39}$

\section{Repetitive TMS}

rTMS involves applying a train of single TMS pulses with a constant frequency and intensity for a given time to the brain target area. It can modulate cortical excitability, with low-frequency rTMS $(\leq 1 \mathrm{~Hz})$ suppressing cortical excitability ${ }^{40}$ and high-frequency rTMS ( $\geq 5 \mathrm{~Hz}$ ) increasing cortical excitability. ${ }^{41}$ rTMS can also be used to noninvasively induce long-lasting modulation of the cortical excitability. The mechanism underlying how rTMS modulates cortical excitability beyond the duration of stimulation is unclear, but LTP and LTD of the cortical synapses are a possible mechanism. Such lasting modulation of cortical activity by rTMS can also be induced in areas outside the motor cortex, and these findings raise the possibility of therapeutic applications of rTMS.

\section{CLINICAL APPLICATIONS IN DEMENTIA}

\section{Diagnostic and prognostic applications of TMS in de- mentia}

Recent studies have suggested that TMS could be a novel tool for investigating the early features of synaptic dysfunc- 
tion in AD patients and the identification of biomarkers. $3,29,42$ The initial AD pathological changes were considered to be the aggregation and accumulation of amyloid-beta and tau proteins that damaged neuron synapses long before the presentation of clinical dementia. These toxic oligomeric species impair synaptic transmission, which could be more precisely correlated with the severity of the disease and an additional cognitive decline related to $\mathrm{AD} .^{3} \mathrm{AD}$ patients show different MTs and SAls at different stages of the disease. A lower RMT, which reflects hyperexcitability of the motor cortex, presents in $\mathrm{MCl}$ and the early stage of AD. The RMT decreases with disease progression, and at an advanced stage, it reduces further with global cortical atrophy of the brain. ${ }^{26}$ Thus, TMS parameters well reflect the functional changes in synapses according to the state in AD patients.

The characteristics of TMS parameters differ among several types of dementia. ${ }^{43}$ In FTD, TMS has revealed central motor circuit abnormalities through reduced-amplitude or absent MEPs, and increased latency of MEPs and the central motor conduction time, even in the absence of clinical pyramidal involvement. ${ }^{31}$ Changes in cortical excitability (RMT), central cholinergic activity (SAI), or intracortical inhibition/ facilitation were not observed in FTD. ${ }^{43,44}$ Pierantozzi et al. ${ }^{45}$ reported that early-onset $A D$ patients showed significant low SICI (ISI = 2-3 ms), whereas FTD patients did not.

Based on the above observations, some studies have investigated using TMS to differentiate AD patients from healthy controls and those with other types of dementia. One study used TMS-based assessment of GABAergic and cholinergic neurotransmission to distinguish patients with AD from FTD patients and healthy controls. ${ }^{42}$ AD showed significant impairment of SAI, and FTD showed dysfunction of SICI-ICF. These findings had high sensitivity and specificity in differentiating patients with each disease from healthy controls. Another multicenter study found that $A D$ and DLB patients showed low SAl, representation of cholinergic deficits, and that FTD and DLB had an abnormality of SICI-ICF associated with GABAergic and glutamatergic circuits. ${ }^{46}$ Moreover, $\mathrm{LICl}$ was most impaired in FTD. These results are related to the biological pathology of neurotransmitters in each neurodegenerative disease, and demonstrate the ability to use TMS parameters to differentiate the diseases with high sensitivity and specificity (Table 1).

It has been suggested that the SAI measure could be used as a tool to identify $\mathrm{MCl}$ patients with an increased risk of converting to dementia. ${ }^{47}$ In studies of $\mathrm{MCl}$ patients and healthy controls, SAI was reduced in multiple-domains amnestic $\mathrm{MCl}$, which has the highest risk of converting to

Table 1. Summary of abnormalities of TMS outcome parameters in dementia

\begin{tabular}{|c|c|c|c|c|c|c|}
\hline \multirow[b]{2}{*}{ Disease } & RMT & MEP & SAI & SICI & SICI-ICF & \multirow{2}{*}{$\begin{array}{c}\text { CSP duration } \\
\text { Activation of inhibitory } \\
\text { cortical interneurons } \\
\text { (GABA-B) }\end{array}$} \\
\hline & \multicolumn{2}{|c|}{$\begin{array}{l}\text { Integrity of corticospinal pathway, } \\
\text { cortical excitability }\end{array}$} & $\begin{array}{l}\text { Central cholinergic } \\
\text { activity }\end{array}$ & \multicolumn{2}{|c|}{$\begin{array}{l}\text { SICI-ICF represents inhibitory } \\
\text { (GABA-A)/facilitatory (NMDA) } \\
\text { intercortical phenomena }\end{array}$} & \\
\hline$A D$ & $\downarrow^{26,27,37,44}$ & $\begin{array}{l}\text { Amplitude } \uparrow^{26} \\
\text { latency }^{27}\end{array}$ & $\downarrow^{37,44,45}$ & $\downarrow^{45}$ & $N^{42}$ & $\begin{array}{c}\uparrow^{27} \\
\text { Advanced stage } \downarrow^{26}\end{array}$ \\
\hline $\mathrm{MCl}$ & $\downarrow^{29,48}$ & - & $\begin{array}{c}\downarrow^{29,37}, \\
(M-a M C l \\
\downarrow^{47}, \\
\text { S-aMCl, NaMCI N N7) }\end{array}$ & $\downarrow^{37}$ & - & - \\
\hline FTD & $N^{31,43,44}$ & $\begin{array}{l}\text { Amplitude } \uparrow^{31,43} \\
\text { latency }^{31}\end{array}$ & $N^{43-45}$ & $\begin{array}{c}\mathrm{N}^{45} \\
\left(\text { PNFA }^{31}\right. \\
\left.\text { SD, bvFTD N }{ }^{31}\right)\end{array}$ & Impaired ${ }^{42,46}$ & - \\
\hline DLB & $N^{38}$ & $N^{38}$ & $\downarrow^{38,39}$ & $N^{38}$ & - & - \\
\hline
\end{tabular}

TMS, transcranial magnetic stimulation; RMT, resting motor threshold; MEP, motor evoked potential; SAl, short-latency afferent inhibition; SICI, short-interval intracortical inhibition; ICF, intracortical facilitation; CSP, contralateral silent period; GABA, gamma-aminobutyric acid; NMDA, N-methyl-D-aspartate; AD, Alzheimer's disease; $\mathrm{N}$, normal; $\mathrm{MCl}$, mild cognitive impairment; $\mathrm{M}$-aMCl, multiple domain amnestic mild cognitive impairment; $\mathrm{S}$-aMCl, single domain amnestic mild cognitive impairment; NaMCI, non-amnestic mild cognitive impairment; FTD, frontotemporal dementia; PNFA, progressive non-fluent aphasia; SD, semantic dementia; bvFTD, behavior variant frontotemporal dementia; DLB, dementia with lewy body. 


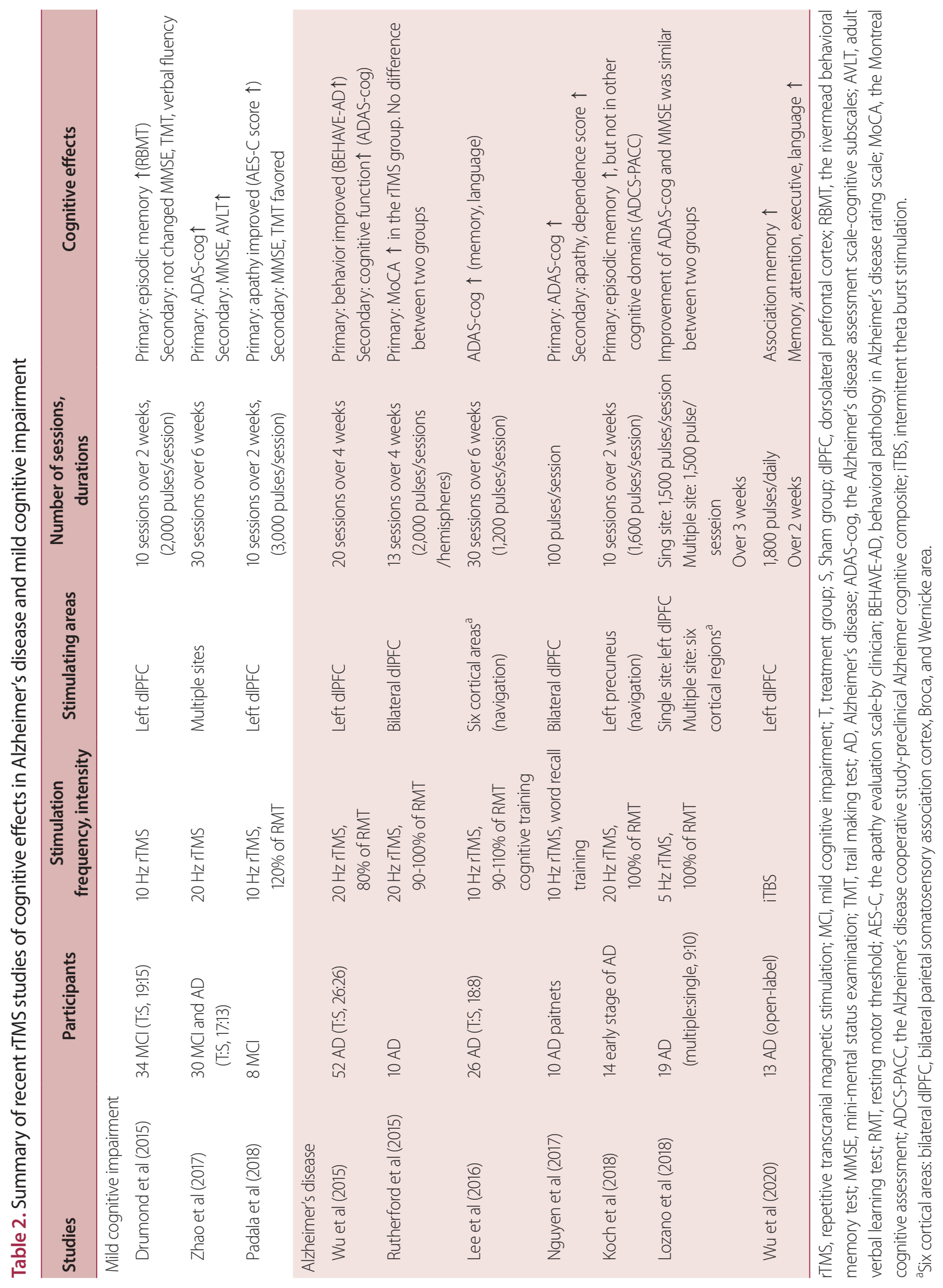


dementia, while it did not differ in single-domain amnestic and non-amnestic $\mathrm{MCl}$ patients compared with normal cognition. ${ }^{47,48}$

One study investigated the benefit of acetylcholinesterase inhibitor (AChEi) treatment in changing plasticity within cortical glutamatergic circuits. ${ }^{49}$ MEP amplitudes were measured during 5-Hz rTMS (which reflects short-term synaptic plasticity) before and after AChEi administration. The application of AChEi for 2 months did not influence the alteration in cortical excitability or short-term synaptic plasticity. Other studies have estimated SAI, which represents central cholinergic activity, and found that the low SAI in AD could be restored by the administration of a single dose of $A C h E i^{37,50}$ Furthermore, AD patients who showed a large increase in SAI after a single dose of AChEi respond well to long-term treatment.

It appears conclusive that the clinical benefit of AChEi treatment is related more closely to factors including cholinergic activity than to the activity of glutamatergic NMDA receptors. Therefore, evaluating SAI could be useful for predicting the response to AChEi.

\section{Therapeutic interventions}

Multiple sessions of rTMS have recently been proposed as a promising treatment for improving cognitive performance in AD (Table 2). rTMS can result in temporal changes in focal cortical excitability producing prolonged changes in neuron integrity. LTP-like cortical plasticity is disrupted in the early stage of AD, whereas high-frequency rTMS can enhance LTPlike cortical plasticity at both the local and network levels. ${ }^{51}$ Multiple sessions of rTMS induced synaptic plasticity and especially LTP, which was the most important mechanism in learning and memory. rTMS was helpful in restoring brain functions and could reflect the potential of rTMS to recruit compensatory networks that underlie memory-encoding and other cognitive processes. ${ }^{52}$

High-frequency rTMS over the dorsolateral prefrontal cortex (dIPFC) can improve global cognition, action naming, object naming, episodic memory, and information processing speed in patients with $A D, M C l$, and cognitively normal controls. ${ }^{53-55}$ Most rTMS studies have focused on the dIPFC, and this area is affected in both the early and late stages of dementia. Researchers have recently applied rTMS to the posterior parietal cortex and precuneus, which were affected in the early stage and had prominent neuropathological abnormalities. The effects of rTMS in various types of dementia are summarized in Fig. 1.

We have reported on the effect of rTMS with cognitive

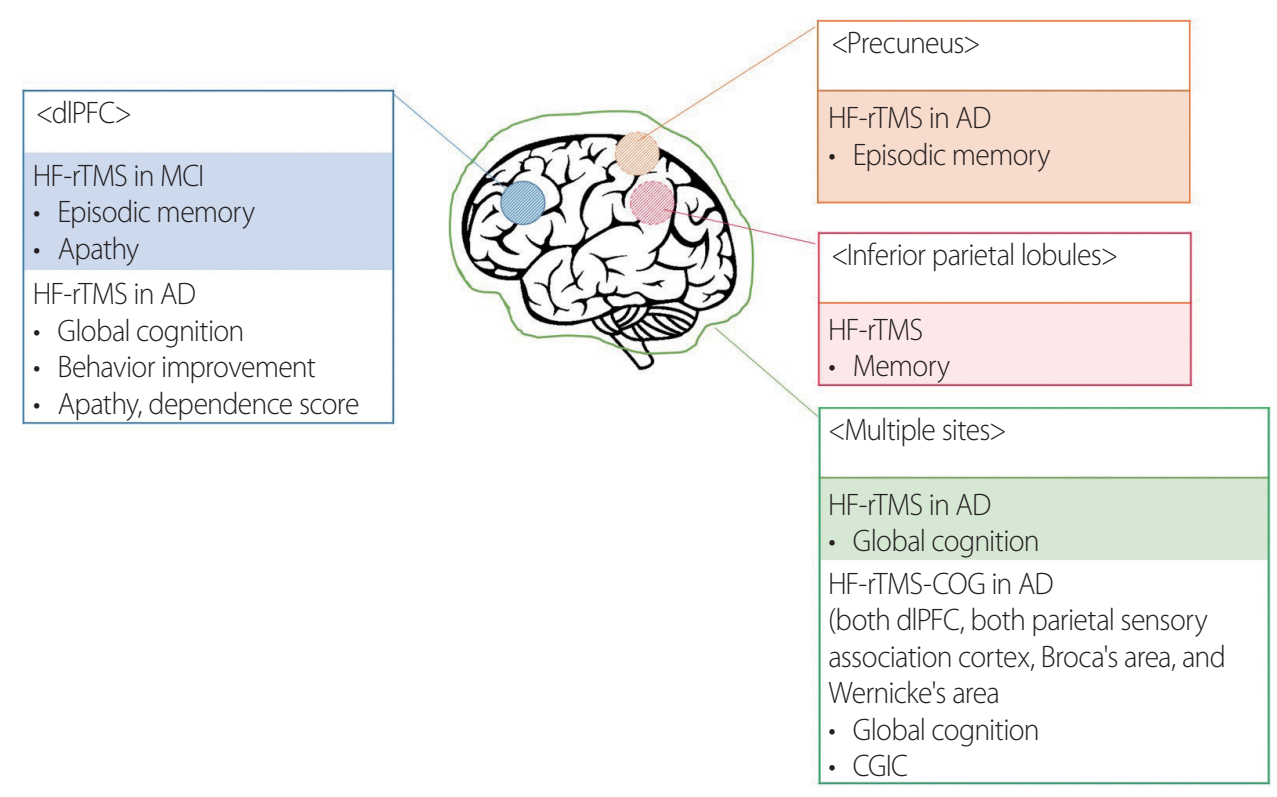

Fig. 1. Effects of high-frequency repetitive transcranial magnetic stimulation (HF-rTMS) in Alzheimer's disease (AD) according to cortical areas. dIPFC, dorsolateral prefrontal cortex; $\mathrm{MCl}$, mild cognitive impairment; $\mathrm{CGIC}$, clinical global impressions of change. 
training (rTMS-CT) in patients with mild-to-moderate $A D .^{56}$ The participants in that study received rTMS in 6 cortical areas and showed cognitive improvement after 30 sessions. The effect was maintained at 6 weeks after the end of the treatment, and was more prominent in the mild-AD group. Similar to our study, other studies have found better cognitive effects of rTMS in the early stage of AD. ${ }^{57}$ These results might be due to the degree of gray-matter atrophy in AD-related brain regions contributing to the variability of rTMS-induced cognitive aftereffects. ${ }^{58}$ Patients with AD who received rTMS-CT showed slower cognitive decline than did those who only took AChEis over 3 years. ${ }^{59}$ That study revealed for how long rTMS-CT could affect the disease course.

rTMS can improve neuropsychiatric symptoms in AD as well as enhance cognitive function. A meta-analysis of the efficacy of rTMS on the neuropsychiatric symptoms of AD found that rTMS could persistently improve the neuropsychiatric symptoms at an early stage after treatment. ${ }^{60}$ However, following the evidence-based guidelines on the therapeutic use of rTMS, ${ }^{61}$ further evidence is needed for the use of rTMS as a therapeutic tool in $\mathrm{AD}$ and $\mathrm{MCl}$.

TBS is emerging as a more efficient treatment paradigm in terms of time and energy than rTMS. ${ }^{62}$ TBS involves applying a $50-\mathrm{Hz}$ triplet pulse burst with a 200-ms interburst interval, typically at $80 \%$ of the active MT. Continuous TBS (CTBS) delivers 600 pulses for 40 seconds without interruption, while intermittent TBS (iTBS) involves 2-s-long TBS trains repeated every 10 seconds for a total of 20 cycles. CTBS generally decreases cortical excitability for up to 1 hour, while iTBS increases cortical excitability more rapidly than conventional rTMS. ${ }^{24}$ Several studies have applied iTBS for treating dementia. In an open-label study, 13 patients with AD received 14 sessions of iTBS daily over a 2-week period. ${ }^{63}$ After treatment, they showed cognitive improvement in memory, attention executive, and language functions, and their behavioral and psychiatric symptoms were also ameliorated.

\section{CONCLUSION}

Many studies have investigated TMS over the last 20 years, and many developments have been made in various fields. TMS paradigms can address many neurophysiological states of the neural integrity and functional changes in neurotransmitters in vivo. It is possible that TMS parameters such as the MT, MEP, SICI-ICF, and SAI could be useful biomarkers for diagnosing and differentiating different types of dementia. Moreover, rTMS and TBS can be adjunctive management methods for cognitive decline and abnormal behavior in AD. Since TMS is a painless, and noninvasive tool, future researches could lead to the development of systematic TMS evaluation and treatment protocols for aiding the diagnosis and management of dementia.

\section{Conflicts of Interest}

The authors declare no conflicts of interest relevant to this article.

\section{REFERENCES}

1. Barker AT, Jalinous R, Freeston IL. Non-invasive magnetic stimulation of human motor cortex. Lancet 1985;1:1106-1107.

2. Pascual-Leone A, Houser CM, Reese K, Shotland LI, Grafman J, Sato $S$, et al. Safety of rapid-rate transcranial magnetic stimulation in normal volunteers. Electroencephalogr Clin Neurophysiol 1993;89:120-130

3. Koch G, Martorana A, Caltagirone C. Transcranial magnetic stimulation: emerging biomarkers and novel therapeutics in Alzheimer's disease. Neurosci Lett 2020;719:134355.

4. Faraday M. Experimental researches in electricity. 1st ed. London: Dover Publications, 1832;1-368.

5. Chervyakov AV, Chernyavsky AY, Sinitsyn DO, Piradov MA. Possible mechanisms underlying the therapeutic effects of transcranial magnetic stimulation. Front Hum Neurosci 2015;9:303.

6. Hallett M. Transcranial magnetic stimulation and the human brain. Nature 2000:406:147-150.

7. Abdeen MA, Stuchly MA. Modeling of magnetic field stimulation of bent neurons. IEEE Trans Biomed Eng 1994;41:1092-1095.

8. Radman T, Ramos RL, Brumberg JC, Bikson M. Role of cortical cell type and morphology in subthreshold and suprathreshold uniform electric field stimulation in vitro. Brain Stimul 2009;2:215228.e3.

9. Thickbroom GW. Transcranial magnetic stimulation and synaptic plasticity: experimental framework and human models. Exp Brain Res 2007;180:583-593.

10. Reato D, Rahman A, Bikson M, Parra LC. Low-intensity electrical 
stimulation affects network dynamics by modulating population rate and spike timing. J Neurosci 2010;30:15067-15079.

11. Peterchev AV, Wagner TA, Miranda PC, Nitsche MA, Paulus W, Lisanby SH, et al. Fundamentals of transcranial electric and magnetic stimulation dose: definition, selection, and reporting practices. Brain Stimul 2012;5:435-453.

12. Gustafsson B, Wigström H. Physiological mechanisms underlying long-term potentiation. Trends Neurosci 1988;11:156-162.

13. Christie BR, Kerr DS, Abraham WC. Flip side of synaptic plasticity: long-term depression mechanisms in the hippocampus. Hippocampus 1994:4:127-135.

14. Huang YZ, Chen RS, Rothwell JC, Wen HY. The after-effect of human theta burst stimulation is NMDA receptor dependent. Clin Neurophysiol 2007;118:1028-1032.

15. Lisanby SH, Belmaker RH. Animal models of the mechanisms of action of repetitive transcranial magnetic stimulation (RTMS): comparisons with electroconvulsive shock (ECS). Depress Anxiety 2000;12:178-187.

16. Li X, Qi G, Yu C, Lian G, Zheng H, Wu S, et al. Cortical plasticity is correlated with cognitive improvement in Alzheimer's disease patients after rTMS treatment. Brain Stimul 2021;14:503-510.

17. Meyer B. Handbook of transcranial magnetic stimulation. 1st ed. London: Arnold, 2002;177-184.

18. Roth Y, Amir A, Levkovitz Y, Zangen A. Three-dimensional distribution of the electric field induced in the brain by transcranial magnetic stimulation using figure-8 and deep H-coils. J Clin Neurophysiol 2007;24:31-38.

19. Hardwick RM, Lesage E, Miall RC. Cerebellar transcranial magnetic stimulation: the role of coil geometry and tissue depth. Brain Stimul 2014;7:643-649.

20. Kakuda W, Abo M, Nakayama Y, Kiyama A, Yoshida H. High-frequency rTMS using a double cone coil for gait disturbance. Acta Neurol Scand 2013;128:100-106.

21. Rubens MT, Zanto TP. Parameterization of transcranial magnetic stimulation. J Neurophysiol 2012;107:1257-1259.

22. Williams JA, Imamura M, Fregni F. Updates on the use of non-invasive brain stimulation in physical and rehabilitation medicine. J Rehabil Med 2009:41:305-311.

23. Modugno N, Nakamura Y, MacKinnon CD, Filipovic SR, Bestmann S, Berardelli A, et al. Motor cortex excitability following short trains of repetitive magnetic stimuli. Exp Brain Res 2001;140:453459.

24. Huang YZ, Edwards MJ, Rounis E, Bhatia KP, Rothwell JC. Theta burst stimulation of the human motor cortex. Neuron
2005;45:201-206.

25. Rossini PM, Barker AT, Berardelli A, Caramia MD, Caruso G, Cracco $R Q$, et al. Non-invasive electrical and magnetic stimulation of the brain, spinal cord and roots: basic principles and procedures for routine clinical application. Report of an IFCN committee. Electroencephalogr Clin Neurophysiol 1994;91:79-92.

26. Alagona G, Bella R, Ferri R, Carnemolla A, Pappalardo A, Costanzo $E$, et al. Transcranial magnetic stimulation in Alzheimer disease: motor cortex excitability and cognitive severity. Neurosci Lett 2001;314:57-60.

27. Khedr EM, Ahmed MA, Darwish ES, Ali AM. The relationship between motor cortex excitability and severity of Alzheimer's disease: a transcranial magnetic stimulation study. Neurophysiol Clin 2011:41:107-113

28. Ferreri F, Pauri F, Pasqualetti P, Fini R, Dal Forno G, Rossini PM. Motor cortex excitability in Alzheimer's disease: a transcranial magnetic stimulation study. Ann Neurol 2003;53:102-108.

29. Mimura Y, Nishida H, Nakajima S, Tsugawa S, Morita S, Yoshida K, et al. Neurophysiological biomarkers using transcranial magnetic stimulation in Alzheimer's disease and mild cognitive impairment: a systematic review and meta-analysis. Neurosci Biobehav Rev 2021;121:47-59.

30. Escudero JV, Sancho J, Bautista D, Escudero M, López-Trigo J. Prognostic value of motor evoked potential obtained by transcranial magnetic brain stimulation in motor function recovery in patients with acute ischemic stroke. Stroke 1998;29:1854-1859.

31. Burrell JR, Kiernan MC, Vucic S, Hodges JR. Motor neuron dysfunction in frontotemporal dementia. Brain 2011;134:2582-2594.

32. Chen R, Lozano AM, Ashby P. Mechanism of the silent period following transcranial magnetic stimulation. Evidence from epidural recordings. Exp Brain Res 1999;128:539-542.

33. Siebner HR, Dressnandt J, Auer C, Conrad B. Continuous intrathecal baclofen infusions induced a marked increase of the transcranially evoked silent period in a patient with generalized dystonia. Muscle Nerve 1998;21:1209-1212.

34. Perretti A, Grossi D, Fragassi N, Lanzillo B, Nolano M, Pisacreta Al, et al. Evaluation of the motor cortex by magnetic stimulation in patients with Alzheimer disease. J Neurol Sci 1996;135:31-37.

35. Ziemann U, Tergau F, Wassermann EM, Wischer S, Hildebrandt J, Paulus W. Demonstration of facilitatory I wave interaction in the human motor cortex by paired transcranial magnetic stimulation. J Physiol 1998;511(Pt 1):181-190.

36. Ziemann U. TMS and drugs. Clin Neurophysiol 2004;115:17171729. 
37. Di Lazzaro V, Oliviero A, Tonali PA, Marra C, Daniele A, Profice P, et al. Noninvasive in vivo assessment of cholinergic cortical circuits in AD using transcranial magnetic stimulation. Neurology 2002:59:392-397.

38. Di Lazzaro V, Pilato F, Dileone M, Saturno E, Profice P, Marra C, et al. Functional evaluation of cerebral cortex in dementia with Lewy bodies. Neuroimage 2007;37:422-429.

39. Marra C, Quaranta D, Profice P, Pilato F, Capone F, lodice F, et al. Central cholinergic dysfunction measured "in vivo" correlates with different behavioral disorders in Alzheimer's disease and dementia with Lewy body. Brain Stimul 2012;5:533-538.

40. Chen R, Classen J, Gerloff C, Celnik P, Wassermann EM, Hallett M, et al. Depression of motor cortex excitability by low-frequency transcranial magnetic stimulation. Neurology 1997;48:1398-1403.

41. Maeda F, Keenan JP, Tormos JM, Topka H, Pascual-Leone A. Interindividual variability of the modulatory effects of repetitive transcranial magnetic stimulation on cortical excitability. Exp Brain Res 2000;133:425-430.

42. Benussi A, Di Lorenzo F, Dell'Era V, Cosseddu M, Alberici A, Caratozzolo $S$, et al. Transcranial magnetic stimulation distinguishes Alzheimer disease from frontotemporal dementia. Neurology 2017:89:665-672.

43. Cantone M, Di Pino G, Capone F, Piombo M, Chiarello D, Cheeran $B$, et al. The contribution of transcranial magnetic stimulation in the diagnosis and in the management of dementia. Clin Neurophysiol 2014;125:1509-1532.

44. Di Lazzaro V, Pilato F, Dileone M, Saturno E, Oliviero A, Marra C, et al. In vivo cholinergic circuit evaluation in frontotemporal and Alzheimer dementias. Neurology 2006;66:1111-1113.

45. Pierantozzi M, Panella M, Palmieri MG, Koch G, Giordano A, Marciani MG, et al. Different TMS patterns of intracortical inhibition in early onset Alzheimer dementia and frontotemporal dementia. Clin Neurophysiol 2004;115:2410-2418.

46. Benussi A, Grassi M, Palluzzi F, Koch G, Di Lazzaro V, Nardone R, et al. Classification accuracy of transcranial magnetic stimulation for the diagnosis of neurodegenerative dementias. Ann Neurol 2020;87:394-404.

47. Nardone R, Bergmann J, Christova M, Caleri F, Tezzon F, Ladurner $\mathrm{G}$, et al. Short latency afferent inhibition differs among the subtypes of mild cognitive impairment. J Neural Transm (Vienna) 2012;119:463-471.

48. Trebbastoni A, Pichiorri F, D'Antonio F, Campanelli A, Onesti E, Ceccanti $\mathrm{M}$, et al. Altered cortical synaptic plasticity in response to $5-\mathrm{Hz}$ repetitive transcranial magnetic stimulation as a new electrophysiological finding in amnestic mild cognitive impairment converting to Alzheimer's disease: results from a 4-year prospective cohort study. Front Aging Neurosci 2016;7:253.

49. Trebbastoni A, Gilio F, D'Antonio F, Cambieri C, Ceccanti M, de Lena $C$, et al. Chronic treatment with rivastigmine in patients with Alzheimer's disease: a study on primary motor cortex excitability tested by $5 \mathrm{~Hz}$-repetitive transcranial magnetic stimulation. Clin Neurophysiol 2012;123:902-909.

50. Di Lazzaro V, Oliviero A, Pilato F, Saturno E, Dileone M, Marra C, et al. Neurophysiological predictors of long term response to AChE inhibitors in AD patients. J Neurol Neurosurg Psychiatry 2005;76:1064-1069.

51. Koch G, Di Lorenzo F, Bonnì S, Ponzo V, Caltagirone C, Martorana A. Impaired LTP- but not LTD-like cortical plasticity in Alzheimer's disease patients. J Alzheimers Dis 2012;31:593-599.

52. Rossi S, Rossini PM. TMS in cognitive plasticity and the potential for rehabilitation. Trends Cogn Sci 2004:8:273-279.

53. Cotelli M, Manenti R, Cappa SF, Zanetti O, Miniussi C. Transcranial magnetic stimulation improves naming in Alzheimer disease patients at different stages of cognitive decline. Eur J Neurol 2008;15:1286-1292.

54. Haffen E, Chopard G, Pretalli JB, Magnin E, Nicolier M, Monnin J, et al. A case report of daily left prefrontal repetitive transcranial magnetic stimulation (rTMS) as an adjunctive treatment for Alzheimer disease. Brain Stimul 2012;5:264-266.

55. Guse B, Falkai P, Wobrock T. Cognitive effects of high-frequency repetitive transcranial magnetic stimulation: a systematic review. J Neural Transm (Vienna) 2010;117:105-122.

56. Lee J, Choi BH, Oh E, Sohn EH, Lee AY. Treatment of Alzheimer's disease with repetitive transcranial magnetic stimulation combined with cognitive training: a prospective, randomized, double-blind, placebo-controlled study. J Clin Neurol 2016;12:57-64.

57. Nardone R, Tezzon F, Höller Y, Golaszewski S, Trinka E, Brigo F. Transcranial magnetic stimulation (TMS)/repetitive TMS in mild cognitive impairment and Alzheimer's disease. Acta Neurol Scand 2014;129:351-366.

58. Anderkova L, Eliasova I, Marecek R, Janousova E, Rektorova I. Distinct pattern of gray matter atrophy in mild Alzheimer's disease impacts on cognitive outcomes of noninvasive brain stimulation. J Alzheimers Dis 2015:48:251-260.

59. Lee J, Sohn EH, Oh E, Song CJ, Jeong SH, Lee AY. Cognitive effect of repetitive transcranial magnetic stimulation with cognitive training: long-term mitigation neurodegenerative effects of mild Alzheimer's disease. Int J Gerontol 2020;14:133-137. 
60. Wang X, Mao Z, Yu X. The role of noninvasive brain stimulation for behavioral and psychological symptoms of dementia: a systematic review and meta-analysis. Neurol Sci 2020;41:1063-1074.

61. Lefaucheur JP, Aleman A, Baeken C, Benninger DH, Brunelin J, Di Lazzaro $V$, et al. Evidence-based guidelines on the therapeutic use of repetitive transcranial magnetic stimulation (rTMS): An update (2014-2018). Clin Neurophysiol 2020;131:474-528.

62. Chu HT, Cheng CM, Liang CS, Chang WH, Juan CH, Huang YZ, et al. Efficacy and tolerability of theta-burst stimulation for major depression: a systematic review and meta-analysis. Prog Neuropsychopharmacol Biol Psychiatry 2021;106:110168.

63. Wu X, Ji GJ, Geng Z, Zhou S, Yan Y, Wei L, et al. Strengthened theta-burst transcranial magnetic stimulation as an adjunctive treatment for Alzheimer's disease: an open-label pilot study. Brain Stimul 2020;13:484-486. 